\title{
On the Three-Parameter Weibull Distribution Shape Parameter Estimation
}

\author{
Mahdi Teimouri ${ }^{1,2}$ and Arjun K. Gupta ${ }^{3 *}$ \\ ${ }^{1}$ Amirkabir University of Technology, ${ }^{2}$ Gonbad Kavous University \\ and ${ }^{3}$ Bowling Green State University
}

\begin{abstract}
The Weibull distribution has received much interest in reliability theory. The well-known maximum likelihood estimators (MLE) of this family are not available in closed form expression. In this work, we propose a consistent and closed form estimator for shape parameter of three-parameter Weibull distribution. Apart from high degree of performance, the derived estimator is location and scale-invariant.
\end{abstract}

Key words: Coefficient of variation, maximum likelihood estimate, Weibull distribution.

\section{Introduction}

The use of Weibull distribution to describe real phenomena has a long history. This distribution was originally proposed by the Swedish physicist Waloddi Weibull. He used it for modeling the distribution of breaking strength of materials. Since then it has received applications in many areas. For a comprehensive review of applications, we refer the readers to Johnson et al. (1994) and Murthy et al. (2004).

The probability density function and the cumulative distribution function of a three-parameter Weibull random variable, say $X$, are:

$$
f_{X}(x)=\frac{\alpha}{\beta}\left(\frac{x-\mu}{\beta}\right)^{\alpha-1} e^{-\left(\frac{x-\mu}{\beta}\right)^{\alpha}},
$$

and

$$
F_{X}(x)=1-e^{-\left(\frac{x-\mu}{\beta}\right)^{\alpha}}
$$

${ }^{*}$ Corresponding author. 
respectively, for $x>\mu, \alpha>0, \beta>0$. The parameters, $\alpha, \beta$ and $\mu$ are known as the shape, scale and location parameters, respectively. The hazard rate function corresponding to (1.1) and (1.2) is

$$
H(x)=\frac{\alpha}{\beta}\left(\frac{x-\mu}{\beta}\right)^{\alpha-1},
$$

for $x>\mu$. So, the Weibull distribution can allow for decreasing, constant and increasing hazard rates. This is one of the attractive properties that made the Weibull distribution so applicable. The non-central moments corresponding to (1.1) and (1.2) are given by

$$
E\left(X^{r}\right)=\sum_{i=0}^{r}\left(\begin{array}{c}
r \\
i
\end{array}\right) \mu^{i} \beta^{r-i} \Gamma\left(1+\frac{r-i}{\alpha}\right),
$$

where $r>i-\alpha$ and $\Gamma(\cdot)$ denotes the gamma function.

Many estimation methods have been proposed for estimating the parameters of the Weibull distribution. We mention: maximum likelihood estimation (Sirvanci and Yang, 1984), moments estimation (Cohen et al., 1984; Cran, 1988), Bayesian estimation (Tsionas, 2000), quantile estimation (Wang and Keats, 1995), logarithmic moment estimation (Johnson et al., 1994) and the probability weighted moment estimation (Bartolucci et al., 1999). The most popular and the most efficient of these is the maximum likelihood estimation.

\section{Main Results}

Among mentioned inference methods, as the most efficient one which received a lot of attention in the literature, MLE of Weibull family is derived by solving the non-linear set of three equations given as follows:

$$
\begin{aligned}
& \frac{n}{\alpha}+\sum_{i=1}^{n} \log \left(\frac{x_{i}-\mu}{\beta}\right)-\sum_{i=1}^{n}\left(\frac{x_{i}-\mu}{\beta}\right)^{\alpha} \log \left(\frac{x_{i}-\mu}{\beta}\right)=0, \\
&-\frac{n \alpha}{\beta}+\frac{\alpha}{\beta} \sum_{i=1}^{n}\left(\frac{x_{i}-\mu}{\beta}\right)^{\alpha}=0, \\
&-(\alpha-1) \sum_{i=1}^{n} \frac{1}{x_{i}-\mu}+\frac{\alpha}{\beta} \sum_{i=1}^{n}\left(\frac{x_{i}-\mu}{\beta}\right)^{\alpha-1}=0 .
\end{aligned}
$$

These equations do not yield closed form solutions for parameters.

Theorem 2.1 is useful for constructing a simple, consistent and closed form estimator for $\alpha$. This estimator is independent of $\beta$. 
Theorem 2.1. Suppose $x_{1}, x_{2}, \cdots, x_{n}$ is a random sample from (1.1). Let $\rho$ denote the sample correlation between the $x_{i}$ and their ranks. Let $C V$ and $S$, respectively, denote the sample coefficient of variation and sample standard deviation. Then,

$$
\rho=\left(\frac{\mu_{X}-\mu}{\sigma_{X}}\right)\left(\frac{1}{2}-\frac{1}{2^{1+1 / \alpha}}\right) \sqrt{\frac{12(n-1)}{(n+1)}} .
$$

Proof. From Stuart (1954), the correlation between $X_{i}$, and their ranks, say $R_{i}$, is:

$$
\rho=\operatorname{corr}\left(X_{i}, R_{i}\right)=\left(\int_{-\infty}^{\infty} x F_{X}(x) d F_{X}(x)-\frac{\mu_{X}}{2}\right) \sqrt{\frac{12(n-1)}{\sigma_{X}^{2}(n+1)}},
$$

where $\mu_{X}=E(X)$ and $\sigma_{X}^{2}=\operatorname{var}(X)$. Using (1.1) and (1.2), we have:

$$
\begin{aligned}
& \int_{\mu}^{\infty} x F_{X}(x) d F_{X}(x) \\
= & \frac{\alpha}{\beta} \int_{\mu}^{\infty} x\left\{1-\exp \left[-\left(\frac{x-\mu}{\beta}\right)^{\alpha}\right]\right\}\left(\frac{x-\mu}{\beta}\right)^{\alpha-1} \exp \left[-\left(\frac{x-\mu}{\beta}\right)^{\alpha}\right] d x \\
= & \frac{\alpha}{\beta} \int_{0}^{\infty}(z+\mu)\left\{1-\exp \left[-\left(\frac{z}{\beta}\right)^{\alpha}\right]\right\}\left(\frac{z}{\beta}\right)^{\alpha-1} \exp \left[-\left(\frac{z}{\beta}\right)^{\alpha}\right] d z \\
= & \frac{\alpha}{\beta} \int_{0}^{\infty}(z+\mu)\left(\frac{z}{\beta}\right)^{\alpha-1} \exp \left[-\left(\frac{z}{\beta}\right)^{\alpha}\right] d z \\
& -\frac{\alpha}{\beta} \int_{0}^{\infty}(z+\mu)\left(\frac{z}{\beta}\right)^{\alpha-1} \exp \left[-2\left(\frac{z}{\beta}\right)^{\alpha}\right] d z \\
= & I_{1}+I_{2}
\end{aligned}
$$

where

$$
I_{1}=\frac{\alpha}{\beta} \int_{0}^{\infty}(z+\mu)\left(\frac{z}{\beta}\right)^{\alpha-1} \exp \left[-\left(\frac{z}{\beta}\right)^{\alpha}\right] d z=\mu+\beta \Gamma(1+1 / \alpha),
$$

and

$$
I_{2}=-\frac{\alpha}{\beta} \int_{0}^{\infty}(z+\mu)\left(\frac{z}{\beta}\right)^{\alpha-1} \exp \left[-2\left(\frac{z}{\beta}\right)^{\alpha}\right] d z=-\frac{\beta \Gamma(1+1 / \alpha)}{2^{1+1 / \alpha}}-\frac{\mu}{2} .
$$

So,

$$
\begin{aligned}
& \int_{\mu}^{\infty} x F_{X}(x) d F_{X}(x)-\frac{\mu_{X}}{2}=I_{1}+I_{2}-\frac{\mu_{X}}{2} \\
& =\beta \Gamma(1+1 / \alpha)\left(\frac{1}{2}-\frac{1}{2^{1+1 / \alpha}}\right)=\left(\mu_{X}-\mu\right)\left(\frac{1}{2}-\frac{1}{2^{1+1 / \alpha}}\right) .
\end{aligned}
$$


Now, divide and simultaneously multiply by, respectively, $\sigma_{X}$ and $\sqrt{\frac{12(n-1)}{n+1}}$ the both sides of (2.8) to see the result.

Corollary 2.1. Suppose $x_{1}, x_{2}, \cdots, x_{n}$ is a random sample from (1.1) with known location parameter. Let $\rho$ denote the sample correlation between the $x_{i}$ and their ranks. Let $C V$ and $S$, respectively, denote the sample coefficient of variation and sample standard deviation. Then an estimator for the shape parameter, $\alpha$ is:

$$
\hat{\alpha}=\frac{-\ln 2}{\ln \left[1-\frac{\rho}{\sqrt{3}}\left(\frac{1}{C V}-\frac{\mu}{S}\right)^{-1} \sqrt{\frac{n+1}{n-1}}\right]} .
$$

From Johnson et al. (1994, p. 656), in some certain cases, it is well known that $X_{(1)}=\min \left\{X_{1}, X_{2}, \cdots, X_{n}\right\}$ is MLE for $\mu$. Generally, this statistic is a consistent estimator of location parameter (see Kundu and Raqab, 2009). A better estimate is $X_{(1)}-1 / n$ (see Sirvanci and Yang, 1984, p. 74). We take the latter statistic as an estimator of the unknown parameter $\mu$, i.e., we let $\hat{\mu}=X_{(1)}-1 / n$. It can be used for constructing a new $\mu$ and $\beta$-independent estimator of shape parameter, $\alpha$ as follows.

Corollary 2.2. Suppose $x_{1}, x_{2}, \cdots, x_{n}$ is a random sample from (1.1) with unknown location parameter. Let $\rho$ denote the sample correlation between the $x_{i}$ and their ranks. Let $C V, S$ and $X_{(1)}$, respectively, denote the sample coefficient of variation, sample standard deviation and minimum statistic. Then a new estimator for the shape parameter, $\alpha$ is:

$$
\hat{\alpha}=\frac{-\ln 2}{\ln \left[1-\frac{\rho}{\sqrt{3}}\left(\frac{1}{C V}-\frac{X_{(1)}-1 / n}{S}\right)^{-1} \sqrt{\frac{n+1}{n-1}}\right]} .
$$

\section{Performance Analysis}

Here we analyze the performance of the new estimator given in (2.10). For the sake of simplicity, let us to consider two cases: $1-\mu$ is known and $2-\mu$ is unknown.

Case 1: In this case, after subtracting $\mu$ from all the points of data set, the problem is reduced to estimating the shape parameter of two-parameter Weibull distribution. Now the new estimator (2.9) depends only on the $C V$ statistic and is:

$$
\hat{\alpha}=\frac{-\ln 2}{\ln \left[1-\frac{\rho}{\sqrt{3}} C V \sqrt{\frac{n+1}{n-1}}\right]} .
$$


Therefore, performance of the new estimator can be discussed by analyzing the sample coefficient of variation. The use and applications of the sample coefficient of variation have a long history (He and Oyadihi, 2001; Tian, 2005). For example, the difference between two populations can be tested by comparing the two sample coefficients of variation based on independent samples gathered from the populations. Due to this importance, tests for the coefficient of variation have been developed (Gupta and Ma, 1996). An approximate $(1-\alpha) \times 100 \%$ confidence interval for $\beta^{2}=\sigma^{2} /|\mu|^{2}$ is

$$
\left[0,\left(\frac{\left(1+b^{2}\right) \chi_{n-1,1-\alpha}^{2}}{n b^{2}}-1\right)^{-1}\right],
$$

where $b=S /|\bar{X}|$ and $\chi_{n-1,1-\alpha}^{2}$ denotes the upper $(1-\alpha)$ percentile of a chi-square distribution with $n-1$ degrees of freedom. Under the normality assumption, we have $\operatorname{var}(b)=\beta^{2}\left(1+2 \beta^{2}\right) /(2 n)$. Similar result holds for two-sided inference. So, because of asymptotic unbiasedness, the confidence interval in (3.2) becomes degenerate for large sample size.

Without the normality assumption, results like (3.2) are not possible. However, Cramér (1964) pointed out that the variance of $b$ can be given as:

$$
\operatorname{var}(b)=\frac{\mu_{1}^{2}\left(\mu_{4}-\mu_{2}^{2}\right)-4 \mu_{1} \mu_{2} \mu_{3}+4 \mu_{2}^{3}}{4 n \mu_{1}^{4} \mu_{2}},
$$

where $\mu_{r}$ denotes the $r$ th central moment of $b$. If observations are from (1.1) then $b=S / \bar{X}$. Johnson et al. (1994, pp. 633-634) have shown that the variance of $b$ is very small for $\alpha \geq 0.1$ and for all admissible values of $\beta$, so, the new estimator given by (3.1) can be expected to perform well.

Case 2: Unfortunately, it is not straightforward to prove unbiasedness and consistency of new estimator (2.10) in this case. Let us to investigate the properties of the estimator $X_{(1)}-1 / n$ and $S$. These estimator are consistent for $\mu$ and $\sigma_{X}$, respectively. Statistically, the ratios $\left(X_{(1)}-1 / n\right) / S$ and $S / \bar{X}$ converge, respectively, to $\mu / \sigma_{X}$ and $1 / C V$ in probability. In the other word, the $\hat{\alpha}$ given in (2.10) converges in probability to $\alpha$. This guarantees that new estimator works well when a sample size is large.

\subsection{Simulation Study}

Here, we compare the performance of the maximum likelihood estimate and the new estimate for the shape parameter of (1.1). The comparison is based on the mean relative error (MRE) criterion defined by

$$
\operatorname{MRE}=\frac{1}{k} \sum_{i=1}^{k}\left|\frac{\alpha-\hat{\alpha}_{i}}{\alpha}\right|,
$$


where $\hat{\alpha}_{i}$ denotes the value of either new estimator or MLE in ith iteration. First, note that here after we consider that $\mu$ is unknown and we call (2.10) as new estimator.

To establish a comprehensive simulation-based study for measuring the efficiency of new estimator comparison with the MLE, MRE is computed for sample sizes of $100,500,1000$ and 2000 when $\mu$ is set on 5 . Larger values of the MRE correspond to less efficient estimator. Figure 1 displays the MREs for a sample of size $n=100,500,1000$ and 2000 for some levels of $\beta=0.5,5$ and $\mu=0$. The MREs for a sample of size $n=100,500,1000$ and 2000 for some levels of $\beta=$ $0.5,5$ and $\mu=10$ are shown in Figure 2. It should be noted that, here, we set the number of iterations $k$, at 100 .

The following observations can be made from Figures 1 and 2:

1. in all cases the difference between MRE of the New estimator and the MLE is not significant.

2. because the New estimator is scale invariant, totally, difference between MREs of the New estimator and MLE is not subject to the scale parameter.

3. in each row of Figures, when $\beta$ increases from 0.5 to 5 , MREs have no significant changes, totally.

4. in each column of Figures, when $n$ increases from 100 to 2000, MREs decrease with increasing $n$, totally.

5. when $\mu$ increases from 0 to 10, comparing Figures 1 and 2, it turns out that degree of dependence of MREs of two estimators on $\mu$ is negligible.

Further discussions show that both new estimator and MLE behaves the same when bias is considered as criterion. The mode of two estimators occur at origin. Although the MLE has higher peak than the new estimator in origin, but simulations show that the sample range of bias of the New estimator and the MLE are approximately equal. Also, normality of the New estimator is verified even form small sample size (here $n=100$ ). The bias frequency histogram are depicted in Figure 3 for some selected levels of $\alpha$ and $\beta$ when location parameter $\mu$, is set on 10. The histogram is constructed from 500 points, with account taken of the fact that each point is obtained via the MLE or new estimator on the basis of a sample of size 100 generated from (1.1).

\subsection{Examples}

In this subsection, we provide two data sets to show how much the new estimator works well. For this mean, we address the readers to data sets is assumed to be distributed with Weibull law (see Murthy et al., 2004, pp. 83, 100). The data sets are given in Tables 1 and 2 as follows. The results for fitting 

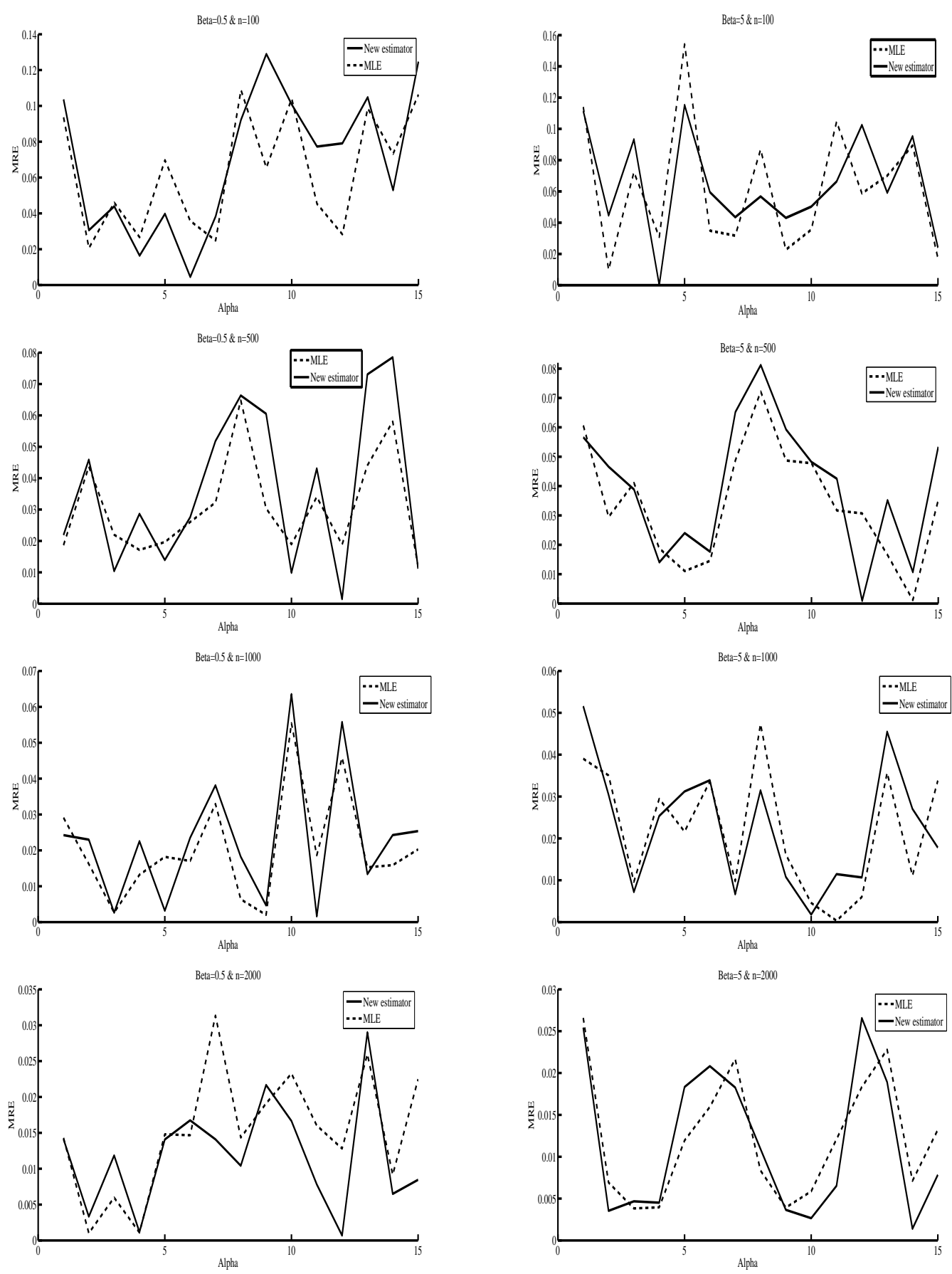

Figure 1: MRE of the New estimator and MLE for some levels of $\alpha$ and $\beta$ when $\mu=0$ 

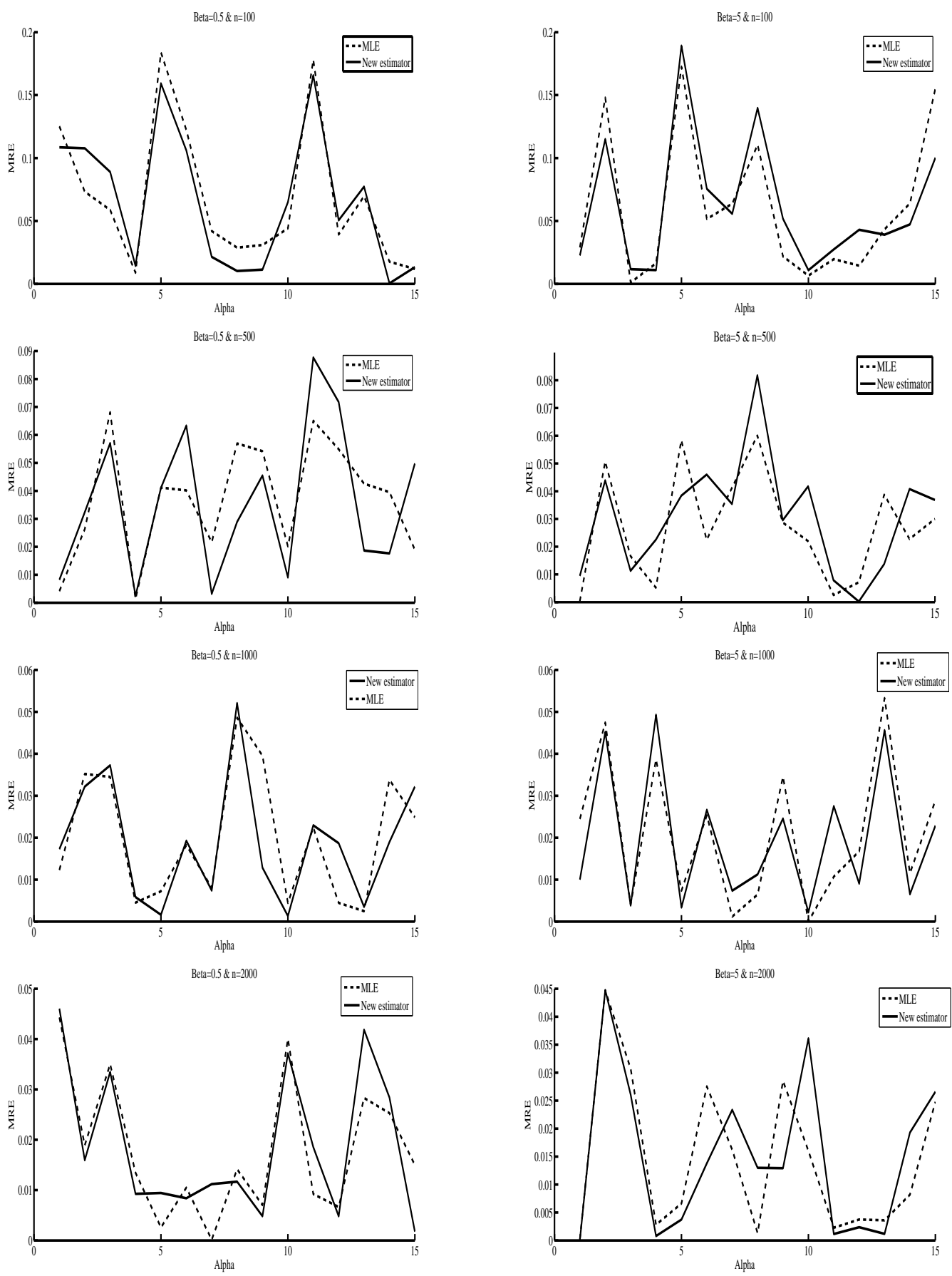

Figure 2: MRE of the New estimator and MLE for some levels of $\alpha$ and $\beta$ when $\mu=10$ 
(MLE)

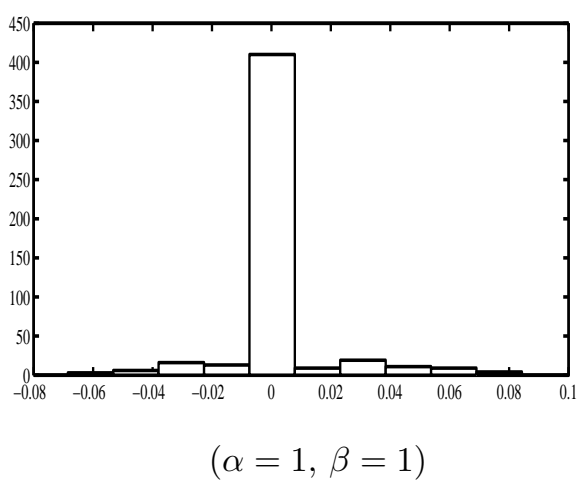

(MLE)

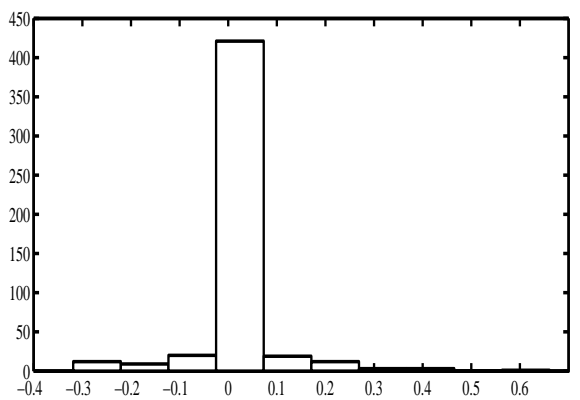

$(\alpha=5, \beta=5)$
(New estimator)

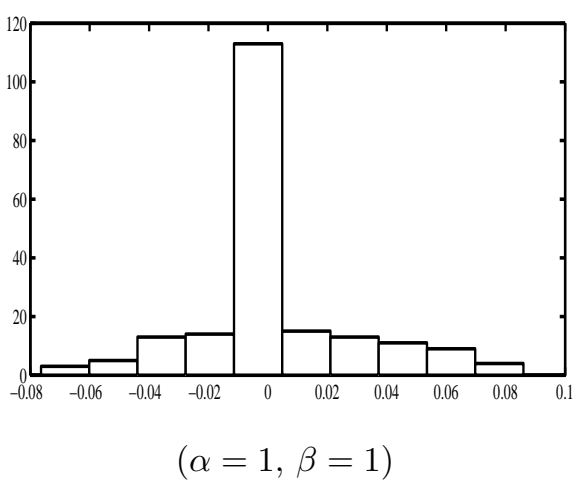

(New estimator)

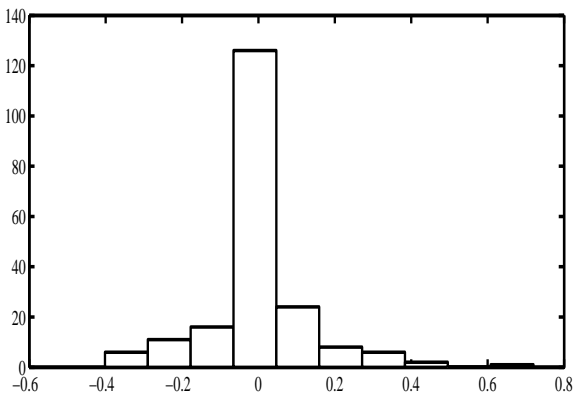

$(\alpha=5, \beta=5)$

Figure 3: Bias frequency of the New estimator and the MLE $\alpha$ for some levels of $\alpha$ and $\beta$

the three-parameter Weibull distribution to the data sets of Tables 1 and 2 are given, respectively, in Tables 3 and 4. It should be noted that, after estimating $\alpha$, $\hat{\beta}$ is estimated through a closed form expression derived by (2.2). Also $X_{(1)}-1 / n$ is considered here as an estimator for $\mu$.

Table 1: Data set 1, failure times of 24 mechanical components

\begin{tabular}{llllllll}
\hline 30.94 & 18.51 & 16.62 & 51.56 & 22.85 & 22.38 & 19.08 & 49.56 \\
17.12 & 10.67 & 25.43 & 10.24 & 27.47 & 14.70 & 14.10 & 29.93 \\
27.98 & 36.02 & 19.40 & 14.97 & 22.57 & 12.26 & 18.14 & 18.84 \\
\hline
\end{tabular}

Table 2: Data set 2, lifetimes of 20 electronic components

\begin{tabular}{llllllllll}
\hline 0.03 & 0.12 & 0.22 & 0.35 & 0.73 & 0.79 & 1.25 & 1.41 & 1.52 & 1.79 \\
1.80 & 1.94 & 2.38 & 2.40 & 2.87 & 2.99 & 3.14 & 3.17 & 4.72 & 5.09 \\
\hline
\end{tabular}


Table 3: Estimated parameters for data set 1

\begin{tabular}{|c|c|c|c|c|}
\hline Method & \multicolumn{3}{|c|}{ Estimated parameters } & $\mathrm{AD}$ distance \\
\hline MLE & $\alpha=1.171$ & $\beta=13.550$ & $\mu=10.100$ & 0.301 \\
\hline New estimator & $\alpha=1.130$ & $\beta=13.294$ & $\mu=10.198$ & 0.378 \\
\hline
\end{tabular}

Table 4: Estimated parameters for data set 2

\begin{tabular}{cccccc}
\hline Method & \multicolumn{3}{c}{ Estimated parameters } & AD distance \\
\cline { 5 - 6 } MLE & $\alpha=1.217$ & $\beta=2.057$ & $\mu=-0.008$ & 0.432 \\
\hline New estimator & $\alpha=1.227$ & $\beta=2.072$ & $\mu=-0.020$ & 0.408 \\
\hline
\end{tabular}

The Anderson-Darling statistic (AD) is given in last column of each table. In the sense of this measure, the MLE works better than the new estimator for data set 1 , while the reverse is concluded for data set 2 from Table 4 .

\section{Conclusion}

In this work a new estimator for shape parameter, as the main parameter, of the three-parameter Weibull family is proposed. Having properties such as: closed form expression, simplicity, asymptotically unbiasedness, high degree of performance and independence of scale and location parameters made it as a good competitor for maximum likelihood estimator of the shape parameter which is computed numerically. Simulations show that the new estimator works comparable with the maximum likelihood estimator, in the sense of (3.4), even for small sample size. Two real examples also verify that the new estimator performs very good.

\section{References}

Bartolucci, A. A., Singh, K. P., Bartolucci, A. D. and Bae, S. (1999). Applying medical survival data to estimate the three-parameter Weibull distribution by the method of probability-weighted moments. Mathematics and Computers in Simulation 48, 385-392.

Cohen, A. C., Whitten, B. J. and Ding, Y. (1984). Modified moment estimation for the three parameter Weibull distribution. Journal of Quality Technology 16, 159-167.

Cramér, H. (1964). Mathematical Methods of Statistics. Princeton University Press, Princeton. 
Cran, G. W. (1988). Moment estimators for the 3-parameter Weibull distribution. IEEE Transactions on Reliability $\mathbf{3 7}, 360-363$.

Gupta, R. C. and Ma, S. (1996). Testing the equality of the coefficient of variation in $k$ normal populations. Communications in Statistics - Theory and Methods 25, 115-132.

He, X. and Oyadiji, S. O. (2001). Application of coefficient of variation in reliability-based mechanical design and manufacture. Journal of Materials Processing Technology 119, 374-378.

Johnson, N. L., Kotz, S. and Balakrishnan, N. (1994). Continuous Univariate Distributions, Volume I, 2nd edition. John Wiley, New York.

Kundu, D. and Raqab, M. Z. (2009). Estimation of $R=P(Y<X)$ for threeparameter Weibull distribution. Statistics and Probability Letters 79, 18391846.

Murthy, D. N. P., Xie, M. and Jiang, R. (2004). Weibull Models. John Wiley, New York.

Sirvanci, M. and Yang, G. (1984). Estimation of the Weibull parameters under type I censoring. Journal of the American Statistical Association 79, 183187.

Stuart, A. (1954). The correlation between variate-values and ranks in samples from a continuous distribution. British Journal of Statistical Psychology 7, $37-44$.

Tian, L. (2005). Inferences on the common coefficient of variation. Statistics in Medicine 2, 2213-2220.

Tsionas, E. G. (2000). Posterior analysis, prediction and reliability in threeparameter Weibull distributions. Communications in Statistics - Theory and Methods 9, 1435-1449.

Wang, F. K. and Keats, J. B. (1995). Improved percentile estimation for the 2-parameter Weibull distribution. Microelectronics and Reliability 35, 883892. 
Mahdi Teimouri

1-Department of Statistics

Faculty of Mathematics \& Computer Science

Amirkabir University of Technology

424, Hafez Ave., Tehran 15914, Iran

teimouri@aut.ac.ir

2-Department of Statistics

Faculty of Science

Gonbad Kavous University

Shahid Fallahi, 163, Gonbad Kavous, Iran

mahdiba_2001@yahoo.com

Arjun K. Gupta

Department of Mathematics \& Statistics

Bowling Green State University

Bowling Green, OH 43403-0221, USA

gupta@bgsu.edu 